\title{
IDENTIFICAÇÃO DE ÁCAROS DA POEIRA DOMICILIAR DE ITUVERAVA-SP
}

CAMPOS, Aline Gomes de ${ }^{1}$

LIPORACI, Tales de Paula Checchia ${ }^{2}$

TERRA, Sílvia Azevedo ${ }^{3}$

Recebido em: 2009.12.12

Aprovado em: 2010.02.22

ISSUE DOI: $10.3738 / 1982.2278-332$

RESUMO: Vários fenômenos alérgicos que acometem o homem podem ser decorrentes do contato com ácaros de poeira domiciliar que são encontrados em residências, as quais disponibilizam nutrição e temperatura e umidade adequadas ao seu desenvolvimento. O objetivo deste estudo foi identificar a fauna acarina da cidade de Ituverava/SP. Foram coletadas amostras de poeira domiciliar em 30 residências do perímetro urbano, selecionadas aleatoriamente para que a amostragem fosse fidedigna em relação à população de ácaros da cidade. Os proprietários dos domicílios responderam a um questionário sobre as características da residência. No momento da coleta aferiu-se a temperatura e a umidade relativa do ar. Em todas as amostras de poeira analisadas encontrou-se ácaros de poeira domiciliar. A principal família observada foi a Pyroglyphidae, com Dermatophagoides farinae, Euroglyphus maynei e Dermatophagoides evansi. A família Cheyletidae foi a segunda mais encontrada. Observou-se também um grande número de ácaros em estádios diferentes de ninfa, demonstrando que estes estavam em período de reprodução. Sugere-se a inclusão de extratos dos ácaros presentes na fauna acarina de Ituverava/SP na bateria de testes cutâneos utilizados na avaliação do paciente com suspeita de alergia respiratória, possibilitando uma adequação dos antígenos empregados nos testes cutâneos para diagnósticos de doenças atópicas.

Palavras-chave: Ácaros de poeira domiciliar; Alergia; Acaro fauna.

\section{IDENTIFICATION OF THE HOUSE DUST MITES IN ITUVERAVA-SP}

SUMMARY: Various allergic phenomena that affect the man may be the result of contact with household dust mites that are found in homes, which provide nutrition and appropriate temperature and humidity for their development. The objective of this study was to identify the acari fauna of the city of Ituverava / SP. Samples were collected from house dust in 30 homes of the urban perimeter, randomly selected for the sampling was reliable for the population of mites in the city. The owners of the households answered a questionnaire on the characteristics of the residence. At the time of collection to the measured temperature and relative humidity. In all samples of dust examined was found house dust mites. The main family observed was The Pyroglyphidae with Dermatophagoides farinae, Euroglyphus maynei and Dermatophagoides evansi. The second family most found was Cheyletidae. There was also a large number of mites in different stages of the development, showing that they were in the reproductive period. We suggest the inclusion of the extracts of the mites in the Acari fauna of Ituverava / SP in the skin tests used to assess the patient with suspected of atopy, allowing an adjustment of the antigens used in skin tests for diagnosis of atopic diseases

Keywords: House dust mites; Allergy; Acari fauna.

\section{INTRODUÇÃO}

A alergia é um estado de hipersensibilidade imunologicamente mediada que resulta da exposição a um alérgeno, o qual é uma substância biológica ou química que causa reações

\footnotetext{
${ }^{1}$ Bióloga - Mestranda em Patologia pela Universidade Federal do Triângulo Mineiro (UFTM)

${ }^{2}$ Biólogo pela Faculdade de Filosofia, Ciências e Letras/FE (FFCL/FE)

${ }^{3}$ Professora Doutora do Curso de Medicina Veterinária da Faculdade Dr. Francisco Maeda/FE (FAFRAM/FE) in memorian
} 
alérgicas (TAKETOMI; SOUZA, 1998). Segundo Gell e Coombs (1975), a hipersensibilidade do tipo I ou imediata é o tipo mais frequente e compreende diversas alterações atópicas como asma, rinite, dermatite atópica, conjuntivite, urticária e reações anafiláticas.

Medeiros Júnior et al. (1997) relataram que os antígenos inaláveis são os mais importantes agentes etiológicos da atopia, dentre os quais se destacam pólens, fungos, poeira domiciliar, ácaros de poeira domiciliar e de estocagem, baratas e epitélio de animais.

A maior parte (2/3) dos fenômenos alérgicos respiratórios do homem são decorrentes não só de ácaros ambientais, como também de seus restos corpóreos e de seus dejetos deixados no ambiente onde o homem habita ou trabalha (BAGGIO, 1989).

Os ácaros de poeira domiciliar são encontrados nas residências, em locais onde se nidificam e reproduzem com facilidade, como travesseiros, colchões, almofadas, tapetes, carpetes, móveis, estofados, roupas de cama, entre outros, desde que estes ambientes ofereçam boas condições ambientais de umidade, temperatura e alimentação. A poeira domiciliar é a camada de partículas suspensas no ar ou que se sedimentam, cobrindo pisos e prateleiras e também aquela que penetrou em colchões, móveis, estofados, carpetes, etc., e em sua composição encontram-se fibras de algodão, lã, descamação da pele humana e de animais, cinzas, pedaços de unhas, fragmentos de pêlos e penas, partículas resultantes do uso normal da casa, grãos de pólen, migalhas de alimentos, etc. (FLECHTMANN, 1986).

Os colchões, travesseiros e almofadas apresentam-se como os melhores locais para o desenvolvimento destes ácaros, destacando-se o colchão, onde o corpo do homem fornece um microambiente ideal, com condições de temperatura, umidade relativa proveniente da transpiração e nutricionais por meio das escamações epidérmicas, que permitem a sobrevivência e reprodução da população de ácaros. Fatores como a temperatura e a umidade relativa são os principais responsáveis pela sua proliferação em determinadas regiões geográficas. Estudos indicam que 100\% da mortalidade de ácaros adultos podem ocorrer com 4 a 11 dias em umidade relativa (UR) abaixo de $40 \%$ e temperaturas abaixo de $25^{\circ} \mathrm{C}$ (GUIMARÃES, 1998).

O conhecimento da fauna acarina pode melhorar os meios de investigação entre a associação e a exposição do alérgeno, podendo assim estabelecer a inclusão de novos extratos de ácaros em testes cutâneos para pacientes com alergia respiratória (SOPELETE et al., 2000; TERRA et al., 2004).

O reconhecimento das espécies de ácaros de poeira domiciliar proporciona um avanço no conhecimento dos possíveis alérgenos da cidade, e, além disso, permite a realização de testes cutâneos mais específicos e diagnósticos mais precisos das alterações atópicas que acometem as vias respiratórias, facilitando o tratamento de ambos e melhorando a qualidade 
de vida do portador da alergia (MEDEIROS JÚNIOR et al., 1997).

O presente estudo teve como objetivo identificar a fauna acarológica existente na poeira domiciliar da cidade de Ituverava - SP, além de aferir a temperatura ambiente e a umidade relativa do ar nos locais de pesquisa.

\section{MATERIAL E MÉTODOS}

Na cidade de Ituverava, situada no nordeste do Estado de São Paulo, com latitude de $20^{\circ} 20^{\prime} 22^{\prime \prime}$ sul, longitude $47^{\circ} 46^{\prime} 50^{\prime \prime}$ oeste e a $631 \mathrm{~m}$ de altitude foram coletadas, durante o verão, em 40 residências, amostras de poeira domiciliar de sofá, cama de casal e cama de solteiro.

A seleção das residências foi feita de forma aleatória para que a amostragem correspondesse à real população dos ácaros de poeira domiciliar ocorrida nesta cidade. Os moradores das residências que concordaram em participar do estudo assinaram um Termo de Consentimento Livre e Esclarecido autorizando a coleta da poeira.

Os sofás, as camas de casal e solteiro constituíram os microambientes nos quais foram coletadas as amostras de poeira domiciliar. Através da aspiração dos estofados e colchões durante cinco minutos por toda a sua superfície, utilizando aspirador de pó portátil, da marca Electrolux (modelo 1300, 250W), ao qual foi acoplado um filtro de papel em sua porção intermediária (marca Jovetta), onde a poeira ficou retida. Estes filtros foram dobrados, identificados, embalados em sacos plásticos e estocados em freezer a $-20^{\circ} \mathrm{C}$ para posterior análise, sem comprometimento dos possíveis ácaros existentes nas poeiras.

Ao trocar de um microambiente para outro, para fazer a aspiração, o tubo coletor do aspirador era lavado com água corrente, detergente e álcool etílico absoluto, sendo seco com papel absorvente.

No momento da coleta de poeira domiciliar foram aferidas a temperatura ambiente e a umidade relativa do ar, utilizando-se termohigrômetro (Hygrotherm, TFA, Alemanha).

Para a análise das amostras de poeira, foi utilizado o método de suspensão, adaptado por Fernandez-Caldas (1997) e a identificação foi realizada através de chaves de identificação, utilizando-se as classificações de Colloff (1992), Perez-Santos (1995) e Fernandez-Caldas (1997). As amostras que com peso inferior a 0,5g de poeira foram descartadas.

Os ácaros foram classificados, sempre que possível, até a espécie e subclassificados com relação ao sexo e, ainda, foram identificados os estádios de ovo e larva. 


\section{RESULTADOS}

Um total de 30 casas foram incluídas neste estudo para a coleta de poeira domiciliar, no entanto, $29(96,67 \%)$ casas apresentaram quantidade de poeira maior que $0,5 \mathrm{~g}$ e $1(3,33 \%)$ apresentou menor que $0,5 \mathrm{~g}$ tendo sido descartada. Foram encontrados ácaros de poeira domiciliar em todas $(100 \%)$ as 29 casas em que as amostras tiveram mais que $0,5 \mathrm{~g}$ de poeira, sendo que estes estiveram presentes em pelo menos uma amostra de cada residência (sofá, cama de casal e cama de solteiro). Assim, foram coletadas poeiras em 29 sofás, 31 camas de casal e 47 camas de solteiro, pois foram coletadas amostras em todas as camas existentes nas casas, totalizando 107 amostras de poeira domiciliar.

Num total de 13 bairros, o bairro Centro foi onde se observou maior concentração de residências de amostras coletadas, num total de 8 (20,00\%), seguido do Jardim Marajoara com $6(15,00 \%)$, da Vila Industrial com 5 (12,50\%), do Jardim Guanabara com 4 (10,00\%) e as demais distribuídas em 9 bairros.

A temperatura e umidade relativas médias de sofás, camas de casal e camas de solteiro foram, respectivamente, representadas (Tabela 1).

Nos sofás foram encontrados 1.012 (100\%) ácaros/g de poeira, sendo que o que apareceu com maior frequência foi o gênero Dermatophagoides, com 714 (70,56\%) ácaros/g de poeira e a espécie Dermatophagoides farinae (Figura 1) com 400 (39,52\%), sendo sua maioria macho $224(22,13 \%)$ (Tabela 2). Aqueles nos quais não foi possível a identificação quanto ao sexo estão representados no somatório total. Foram encontradas 132 (13,04\%) protoninfas, $66(6,52 \%)$ tritoninfas, $50(4,4 \%)$ larvas e $26(2,57 \%)$ ovos. Observou-se que os ácaros do gênero Cheyletus apareceram apenas nas amostras de sofá 18 (1,78\%) (Tabela3).

Nas amostras de poeira da cama de casal foi encontrado um total de 2.982 ácaros/g de poeira, sendo o gênero Dermatophagoides $1.758(58,96 \%)$ o mais frequente, seguido pelo gênero Euroglyphus com as espécies Dermatophagoides farinae (Figura 1) com 1.126 (39,52\%), Dermatophagoides evansi com 174 (5,83\%) e Euroglyphus maynei com 108 $(3,62 \%)$ (Tabela 2). Ainda foram encontradas 454 (15,22\%) protoninfas, 170 (5,70\%) tritoninfas, 352 larvas $(11,80 \%)$ e $72(2,41 \%)$ ovos (Tabela 3$)$. 

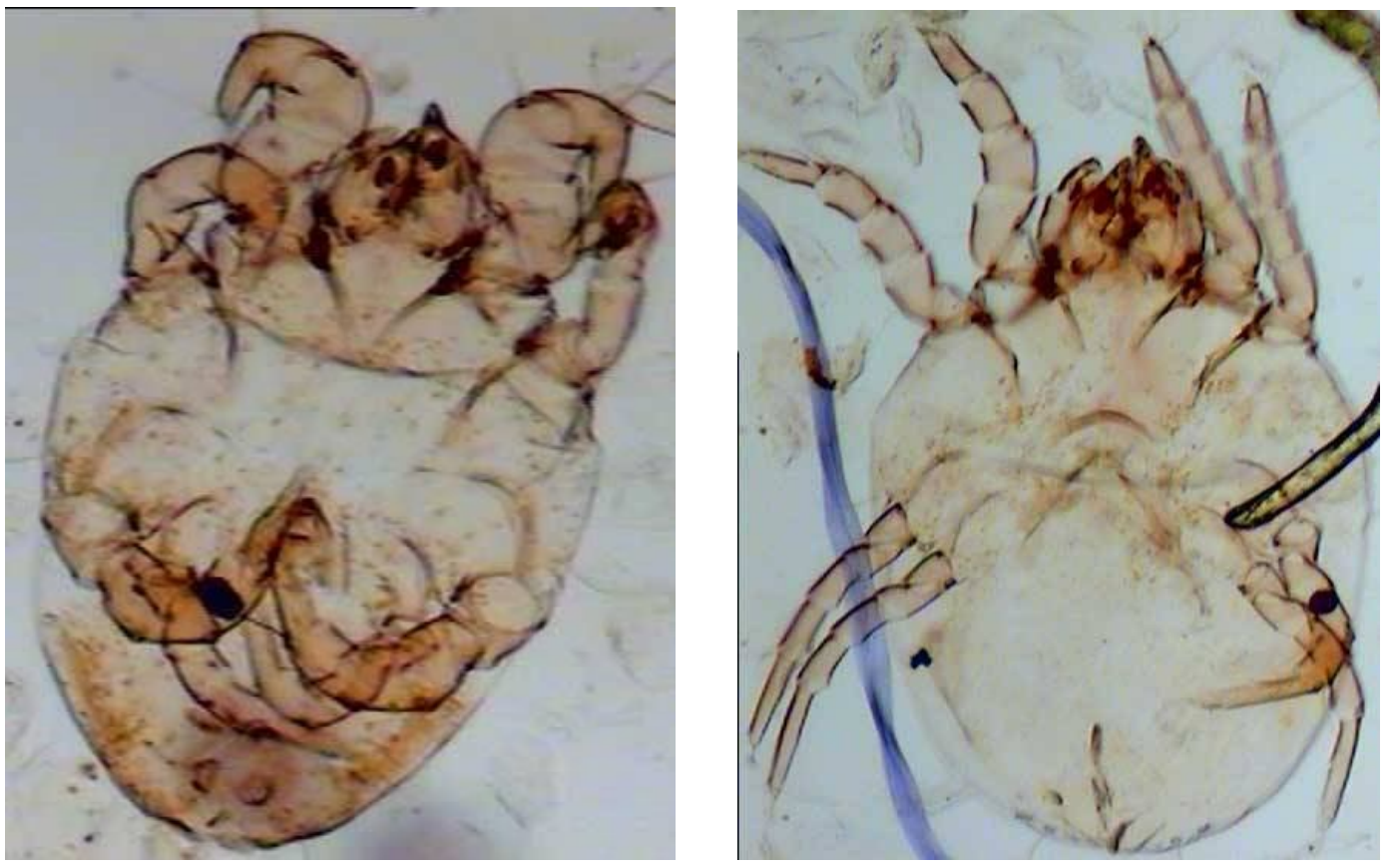

Figura 1: Ácaro da espécie Dermatophagoides farinae, em A - macho e em B - fêmea, 600X.

A

B

Nas camas de solteiro, a quantidade total de ácaros encontrados foi de 5.112 ácaros/g de poeira, sendo o gênero Dermatophagoides 2.762 (54,03\%) o mais frequente, seguido pelo gênero Euroglyphus, com as espécies Dermatophagoides farinae (Figura 1) com 1.666 (32,59\%), Euroglyphus maynei 352 (6,89\%) e Dermatophagoides pteronyssinus com 52 $(1,02 \%)$ (Tabela 2). Ainda foram encontradas 954 (18,66\%) protoninfas, 880 larvas $(17,21 \%)$, $280(5,48 \%)$ tritoninfas e $102(2 \%)$ ovos (Tabela 3$).$

Tabela 1 - Valores de temperatura (T) e umidade relativa (UR), expressos em mediana, nos ambientes das residências em que foram coletadas as amostras de poeira domiciliar na cidade de Ituverava/SP.

\begin{tabular}{lcc} 
Ambientes & Umidade Relativa $(\boldsymbol{\%})$ & Temperatura $\left({ }^{\mathbf{}} \mathbf{C}\right)$ \\
\hline Sala & 59,00 & 30,00 \\
Quarto de Casal & 64,00 & 30,00 \\
Quarto de Solteiro & 63,00 & 29,50 \\
\hline
\end{tabular}


Tabela 2 - Taxonomia, classificação quanto ao sexo e frequência de ácaros encontrados nos sofás, camas de casal e camas de solteiro das residências em que foram coletadas as amostras de poeira domiciliar na cidade de Ituverava/SP.

\section{MICROAMBIENTE}

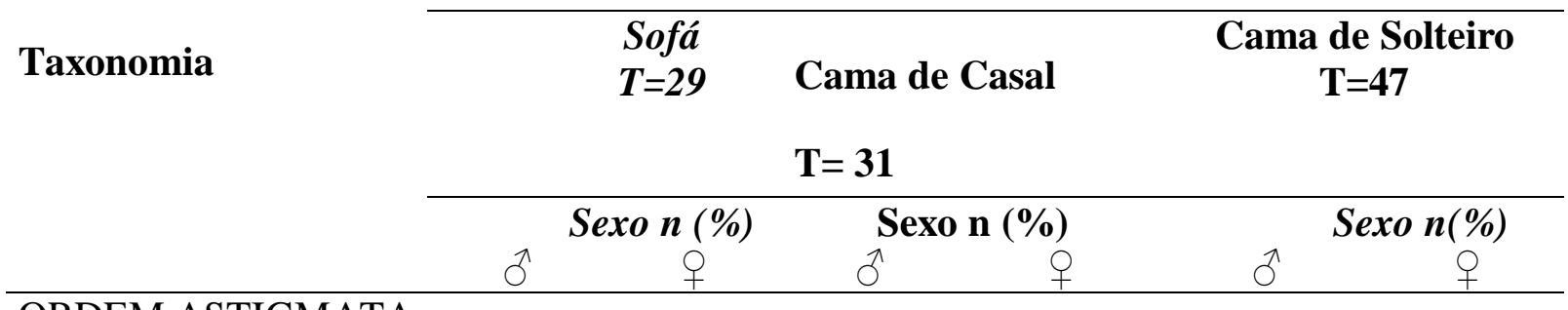

ORDEM ASTIGMATA

Família Acaridae

\begin{tabular}{lllllll}
\hline Acarus siro & $0(0,00)$ & $2(0,20)$ & $0(0,00)$ & $2(0,07)$ & $0(0,00)$ & $0(0,00)$ \\
\hline
\end{tabular}

Família Pyroglyphidae

Gênero

Dermatophagoides

\begin{tabular}{lcccccc}
\hline D. farinae & 224 & 176 & 584 & 542 & 1.076 & 590 \\
& $(22,13)$ & $(17,39)$ & $(19,58)$ & $(18,18)$ & $(21,05)$ & $(11,54)$ \\
\hline D. pteronyssinus & $10(0,99)$ & $12(1,19)$ & $4(0,13)$ & $0(0,00)$ & $44(0,86)$ & $8(0,16)$ \\
\hline D. neotropicalis & $8(0,79)$ & $2(0,20)$ & $2(0,07)$ & $0(0,00)$ & $2(0,04)$ & $0(0,00)$ \\
\hline D. evansi & $14(1,38)$ & $6(0,59)$ & $\begin{array}{c}162 \\
(5,43)\end{array}$ & $12(0,40)$ & $150(2,93)$ & $12(0,23)$ \\
& & & & & \\
\hline
\end{tabular}

Gênero Euroglyphus

\begin{tabular}{lllllll}
\hline Euroglyphus maynei & $2(0,20)$ & $4(0,40)$ & $10(0,34)$ & $88(2,95)$ & $8(0,16)$ & $326(6,38)$ \\
\hline
\end{tabular}

\begin{tabular}{|c|c|c|c|c|c|c|}
\hline $\begin{array}{l}\text { ORDEM } \\
\text { PROSTIGMATA }\end{array}$ & & & & & & \\
\hline Família Cheyletidae & & & & & & \\
\hline Cheyletus sp & $8(0,79)$ & $0(0,00)$ & $4(0,13)$ & $70(2,35)$ & $0(0,00)$ & $12(0,23)$ \\
\hline Total & $\begin{array}{c}266 \\
(26,28)\end{array}$ & $\begin{array}{c}202 \\
(19,97)\end{array}$ & $\begin{array}{c}766 \\
(25,68)\end{array}$ & $\begin{array}{c}714 \\
(23,95)\end{array}$ & $\begin{array}{l}1.280 \\
(25,04)\end{array}$ & $\begin{array}{c}948 \\
18,54)\end{array}$ \\
\hline
\end{tabular}

Onde $\mathrm{n}=$ número de ácaros/g de poeira, $\mathrm{T}=$ total de amostras de poeira analisadas 
Tabela 3 - Taxonomia e frequência de ácaros encontrados nos sofás, camas de casal e camas de solteiro das residências em que foram coletadas as amostras de poeira domiciliar na cidade de Ituverava/SP.

\begin{tabular}{llll}
\hline \multirow{2}{*}{ Taxonomia } & Sofá & Cama de Casal & Cama de Solteiro \\
& $\mathrm{T}=29$ & $\mathrm{~T}=31$ & $\mathrm{~T}=\mathbf{4 7}$ \\
\hline
\end{tabular}

ORDEM ASTIGMATA

Família Acaridae

\begin{tabular}{llll}
\hline Acarus siro & $2(0,20)$ & $2(0,07)$ & $0(0)$ \\
\hline
\end{tabular}

Família Pyroglyphidae

Gênero Dermatophagoides

Dermatophagoides farinae

D. pteronyssinus $400(39,52)$

D. neotropicalis

$22(2,18)$

$10(0,99)$

$20(1,97)$

D. evansi

$254(25,10)$

Dermatophagoides sp

Gênero Euroglyphus

Euroglyphus maynei

$8(0,80)$

\begin{tabular}{rr}
$1.126(37,76)$ & $1.666(32,59)$ \\
$4(0,13)$ & $52(1,02)$ \\
\hline $2(0,07)$ & $2(0,04)$ \\
\hline $174(5,83)$ & $162(3,16)$ \\
\hline $344(11,54)$ & $528(10,33)$ \\
\hline $108(3,63)$ & $352(6,89)$ \\
\hline
\end{tabular}

\section{ORDEM PROSTIGMATA}

Família Cheyletidae

\begin{tabular}{lrrr}
\hline Cheyletus sp & $18(1,78)$ & $170(5,70)$ & $126(2,46)$ \\
\hline Ovos & $26(2,57)$ & $72(2,41)$ & $102(2,00)$ \\
\hline Larvas & $50(4,94)$ & $352(11,80)$ & $880(17,21)$ \\
\hline Protoninfas & $132(13,04)$ & $454(15,22)$ & $954(18,66)$ \\
\hline Tritoninfas & $66(6,52)$ & $170(5,70)$ & $280(5,48)$ \\
\hline
\end{tabular}

\begin{tabular}{llll}
\hline Não identificados & $4(0,40)$ & $4(0,13)$ & $8(0,16)$ \\
\hline
\end{tabular}

\begin{tabular}{llll}
\hline Total & $1.012(100) *$ & $2.982(100)$ & $5.112(100)$ \\
\hline
\end{tabular}

Onde $\mathrm{n}=$ número de ácaros $/ \mathrm{g}$ de poeira, $\mathrm{T}=$ total de amostras de poeira analisadas. ${ }^{*} \mathrm{H}=17,278 ; \mathrm{p}<0,001$

\section{DISCUSSÃO}

No presente estudo, a família Pyroglyphidae foi mais frequente, seguida da Cheyletidae. Segundo Guimarães (1998), Terra et al. (2004) e Binotti et al. (2005), as famílias de ácaros predominantemente encontrados na poeira domiciliar no Brasil são Pyroglyphidae, Glycyphagidae, Acaridae e Cheyletidae, no entanto, neste estudo não foram encontrados exemplares das famílias Glycyphagidae e Acaridae, devido às condições de umidade relativa e temperatura ambiente diferentes das outras regiões estudadas. 
Os ácaros de poeira domiciliar foram encontrados em todas as residências estudadas e o microambiente em que se encontrou maior frequência de ácaros foi a cama de solteiro, provavelmente devido à presença de fatores nutricionais e climáticos favoráveis ao crescimento e proliferação desses microrganismos. Segundo Wickens et al. (1997) os altos níveis de ácaros em poeira domiciliar é devido a uma variedade do estilo de vida e fatores climáticos, além de se observar que famílias com maior número de crianças e de classe social inferior possui um número maior de ácaros nas camas de solteiro, provavelmente devido aos diferentes comportamentos de limpeza e de acordo com Custovic (1996) a idade do colchão não interfere na quantidade de ácaros encontrados.

Observou-se também um elevado percentual de larvas e ninfas nas amostras coletadas, o que sugere a ocorrência de proliferação acarina favorecida por fatores ambientais e climáticos nas residências estudadas.

$\mathrm{Na}$ cidade de Ituverava/SP a temperatura e a umidade relativa podem ter influenciado na prevalência da espécie $D$. farinae, já que a condição climática do ambiente domiciliar interfere no número e na variedade de ácaros encontrados (EZEQUIEL et al., 2001). Em seu estudo Silva et al.(2001) encontrou dentro da fauna acarológica as espécies D. pteronyssinus, D. farinae, E. maynei entre outras espécies ainda não descritas no Brasil, havendo correlação entre as condições climáticas variadas e o número de ácaros. Porém, segundo Arlian et al. (1999), para impedir o crescimento da população de $D$. farinae a umidade relativa deve ser superior a $75 \%$ de 2 a 8 horas por dia e para impedir completamente o crescimento da população, a UR deve ser mantida abaixo de $35 \%$ no mínimo 22 horas por dia e acima de 85\% por 2 horas. Segundo Arlian et al. (1998), a população de E. maynei, não consegue sobreviver em ambientes com umidade relativa acima de $70 \%$ por 12 horas, mas se esta umidade diminuir e a quantidade de alimentos for suficiente, em pouco tempo sua população recupera a estabilidade. De acordo com os fatores biológicos, a variação encontrada em relação ao número de ácaros e entre os ambientes pode ser reflexo de outros fatores como disponibilidade de alimentos, entre outros.

No Peru, onde a umidade relativa é superior a $80 \%$, Croce et al. (2000) em estudos da fauna acarina não encontraram a espécie $D$. farinae.

Segundo Guimarães (1998), em seu trabalho em Salvador/BA, obteve a média da umidade relativa e temperatura mais elevadas do que as encontradas neste trabalho, sendo neste caso, a espécie mais encontrada a D. pteronyssinus. Terra (2001), na cidade de Uberaba/MG também obteve em seu estudo UR mais alta que a da cidade de Ituverava/SP e a espécie predominante também foi $D$. pteronyssinus. 
De acordo com Solarz (2001) a espécie de ácaro mais encontrada na Polônia é o $D$. farinae seguida do E. maynei.

Neste trabalho observou-se a presença do gênero Cheyletus em amostras de poeira domiciliar, demonstrando assim, competição entre as espécies de ácaros, já que se trata de uma espécie de ácaro predador. Esse achado mostra-se condizente com o observado por Guimarães (1998) que demonstrou a presença de Cheyletus sp em suas amostras de poeira domiciliar coletadas em Salvador/BA.

\section{CONCLUSÃO}

Os ácaros da poeira domiciliar estiveram presentes em todas as residências pesquisadas na cidade de Ituverava/SP, sendo que foram encontrados ácaros da família Pyroglyphidae (D. farinae, D. evansi, D. pteronyssinus, D. neotropicalis, Euroglyphus maynei) e da família Cheyletidae (Cheyletus sp), sendo que a espécie predominante foi o $D$. farinae, havendo grande quantidade de ovos e ninfas, demonstrando que os ácaros estavam em período reprodutivo.

Além disso, foi possível transmitir orientação às pessoas e sugerir a adoção de medidas preventivas específicas relacionadas aos cuidados com o ambiente que possam beneficiar o paciente alérgico, com diminuição do absenteísmo escolar e laboral, maior participação em atividades sociais, de lazer e esportivas e diminuição dos gastos com medicamentos, consultas médicas e, eventualmente, despesas hospitalares.

Com estes novos conhecimentos, conclui-se que, se ainda não estiverem presentes, os extratos de $D$. farinae e Cheyletus sp, devem ser incluídos no testes de hipersensibilidade na avaliação do paciente com suspeita de alergia respiratória.

\section{REFERÊNCIAS}

ARLIAN, L. G. et al. Population dynamics of the house dust mites Dermatophagoides farinae, D. pteronyssinus, and Euroglyphus maynei (Acari: Pyroglyphidae) at specific relative humidities. Journal of Medical Entomology, v. 35, n. 1, p. 46-53, 1998.

ARLIAN, L. G.; NEAL, J. S.; VYSZENSKI-MOHER, D. L. Reducing relative humidity to control the house dust mite Dermatophagoides farinae. The Journal of Allergy and Clinical Immunology, v. 104, n. 4, p. 852-856, 1999.

BAGGIO, D. Ácaros da poeira domiciliar e alergia. In: SEMINÁRIOS SOBRE INSETOS E ÁCAROS. Anais... 3. Campinas: Sociedade Entomológica do Brasil Fundação Cargil, 185p. p.173-185, 1989. 
BINOTTI, R. S. et al. Ácaros em poeira de tapetes na cidade de Campinas. Revista Brasileira de Alergia e Imunopatologia, v. 28, n. 1, p. 44-46, Jan/Fev. 2005.

COLLOFF, M. J.; SPIEKSMA, F. T. H. Pictorial keys for identification of domestic mites. Clinical and Experimental Allergy, v. 22. p. 823-830, 1992.

CROCE, M. et al. House dust mites in the city of Lima, Peru. Journal of Investigational Allergology and Clinical Immunology, v10, n. 5, p. 286-288, 2000.

CUSTOVIC, A. et al. New mattresses: how fast do they become a significant source of exposure to house dust mite allergens? Clinical and Experimental Allergy, v. 26, n. 11, p. 1225-1227, nov. 1996.

EZEQUIEL, O. DA S. et al. Evaluation of the acaro fauna of the domiciliary ecosystem in Juiz de Fora, State of Minas Gerais, Brazil. Memórias do Instituto Oswaldo Cruz, v. 96, n. 7, p. 911-916, out. 2001.

FERNANDEZ-CALDAS. Biology and identification of house dust mites. Madrid. 1995. $10 \mathrm{p}$.

FLECHTMANN, C. Ácaros em produtos armazenados e na poeira domiciliar. Piracicaba: FEALQ, 97p., 1986.

GELL, P. H. G.; COOMBS, R. R. A. Classification of allergic reactions responsible for clinical hypersensitive and disease. In: GELL, P. H. G.; COOMBS, R. R. A.; LACHMANN, P. J. Clinical Aspects of Immunology, 3. ed. London. 1975. Cap. 25

GUIMARÃES, K. S. Identificação de ácaros da poeira domiciliar na cidade de SalvadorBA: Estudo preliminar. 1998. 52f. Trabalho de Conclusão de Curso (Graduação em Ciências Biológicas). Universidade Federal da Bahia. Salvador.

MEDEIROS JÚNIOR, M. Sensibilização a aeroalérgenos em indivíduos portadores de asma brônquica e/ou rinite crônica em Salvador-Bahia. 1997. 71f. Dissertação (Mestrado em Medicina) Universidade Federal da Bahia. Salvador.

PÉREZ-SANTOS, C.; ARMENTIA, A. Course on mite identification if families, genera, and species of common house dust and storage food mites. European Congress of Allergology and Clinical Immunology. Madrid.28-30 jun., 1995, 82p.

SILVA, E. et al Evaluation of the acarofauna of the domiciliary ecosystem in Juiz de Fora, State of Minas Gerais, Brazil. Memorial do Instituto Oswaldo Cruz, v. 96, n. 7, p. 911-916, Out. 2001.

SOLARZ, K. Risk of exposure to house dust pyroglyphid mites in Poland. Annals of Agricultural and Environmental Medicine, v. 8, n. 1, p. 11-24, 2001.

SOPELETE, M. C.; SILVA, D. A.; ARRUDA, L. K.; CHAPMAN, M. D.; TAKETOMI, E. A. Dermatophagoides farinae (Der f 1) and Dermatophagoides pteronyssinus (Der p 1) allergen exposure among subjects living in Uberlândia, Brazil. International Archives of Allergy and Immunology, v. 122, n. 4, p. 257-263, Ago. 2000. 
TAKETOMI, E. A.; SOUZA, G. G. Exposição a alérgenos inalantes domiciliares entre pacientes asmáticos de Uberlândia-MG. 1998. 35f. Relatório final para o Cnpq.

Universidade Federal de Uberlândia. Uberlândia, Ago. 1998.

TERRA, S. A. Prevalência de ácaros e suas frações alergênicas em amostras de poeira da cidade de Uberaba-MG. 2001. 99f. Dissertação (Mestrado em Imunologia e Parasitologia Aplicadas) Universidade Federal de Uberlândia.

TERRA, S. A. et al. Mite allergen levels and acarologic analysis in house dust samples in Uberaba, Brazil. Journal of Investigational Allergology and Clinical Immunology.v.14, n. 3, p. 232-237, 2004.

WICKENS, K. et al .Determinants of house dust mites allergen in homes in Wellington, New Zealand. Clinical and Experimental Allergy. v. 27, n. 9, p. 1077-1085, Set. 1997. 
